\title{
Bodiniosides S-Y, Seven New Triterpenoid Saponins from Elsholtzia bodinieri and Their Anti-Influenza Activities
}

\author{
Linyao Yang ${ }^{1,+}$, Jiangchao Du ${ }^{1,+}$, Rongtao $\mathrm{Li}^{1}$, Fei Yu ${ }^{2}$ and Jindong Zhong ${ }^{1, *}$ \\ 1 Faculty of Life Science and Technology, Kunming University of Science and Technology, \\ Kunming 650500, China; linyaoyang0808@163.com (L.Y.); dujiangchao2021@163.com (J.D.); \\ rongtaolikm@163.com (R.L.) \\ 2 Medical School, Kunming University of Science and Technology, Kunming 650500, China; feiyuz8@163.com \\ * Correspondence: jindongzhongkm@163.com or zhongjd@kust.edu.cn; Tel.: +86-871-6591-6009 \\ + These authors contributed equally to this work.
}

check for updates

Citation: Yang, L.; Du, J.; Li, R.; Yu, F.; Zhong, J. Bodiniosides S-Y, Seven New Triterpenoid Saponins from Elsholtzia bodinieri and Their Anti-Influenza Activities. Molecules 2021, 26, 6535. https://doi.org/ $10.3390 /$ molecules 26216535

Academic Editor: Stefano Dall'Acqua

Received: 23 September 2021

Accepted: 26 October 2021

Published: 29 October 2021

Publisher's Note: MDPI stays neutral with regard to jurisdictional claims in published maps and institutional affiliations.

Copyright: (c) 2021 by the authors. Licensee MDPI, Basel, Switzerland. This article is an open access article distributed under the terms and conditions of the Creative Commons Attribution (CC BY) license (https:// creativecommons.org/licenses/by/ $4.0 /)$.
Abstract: Investigation of the $\mathrm{n}-\mathrm{BuOH}$ extract of the aerial parts of Elsholtzia bodinieri led to the isolation of seven new triterpenoid saponins, Bodiniosides S-Y (1-7, resp.). Their strictures were elucidated on the basis of spectroscopic techniques, including HSQC, HSBC, and HSQC-TOCSY experiments, together with acid hydrolysis and GC analysis. The anti-influenza activities of compounds 1-7 were evaluated against A/WSN/33/2009 (H1N1) virus in MDCK cells. The results showed that compounds 2 and 5 exhibited moderate anti-influenza activities against A/WSN/33/2009 (H1N1), with inhibition rates of $35.33 \%$ and $24.08 \%$, respectively.

Keywords: Elsholtzia bodinieri; triterpenoid saponins; anti-influenza virus activities; bodiniosides S-Y

\section{Introduction}

Elsholtzia bodinieri Vaniot (Chinese name "Dongzisu"), belonging to the taxonomically diverse group of the family Labiatae, is a medicinal plant that grows in Yunnan and Guizhou Provinces in China. It is commonly known as "yashuacao" and is used as a traditional Chinese medicine for the treatment of cough, headache, pharyngitis, fever and hepatitis [1]. Previous studies on E. bodinieri led to the isolation of triterpenoid saponins [2-6], flavonoid glycosides [7,8], sesquiterpene glycosides [9], clerodane diterpenoid glycosides [10], and phenolic constituents [11] from the aerial parts of this plant. As a continuation of our work, we further systematically investigated the chemical components of the aerial parts of this plant. In our search for secondary metabolites with structural diversity and potential anti-influenza virus activity, seven new triterpenoid saponins, Bodiniosides S-Y (1-7, resp.), were obtained from E. bodinieri. Among them, compounds $\mathbf{2}$ and $\mathbf{5}$ exhibited moderate inhibition of influenza virus activities with inhibition rates of $35.33 \%$ and $24.08 \%$, respectively. Herein, we report the isolation, structural elucidation and anti-influenza virus activities of the isolated compounds.

\section{Results}

The $\mathrm{n}-\mathrm{BuOH}$ soluble fraction of the $75 \%$ aqueous acetone extract of the aerial parts of E. bodinieri was subjected to repeated column chromatography over silica gel, Sephadex LH-20, RP-18, and semipreparative reversed-phase HPLC, eluting with various solvent systems, to afford seven new triterpenoid saponins (Figure 1). The spectrums can be found in supplementary materials. 


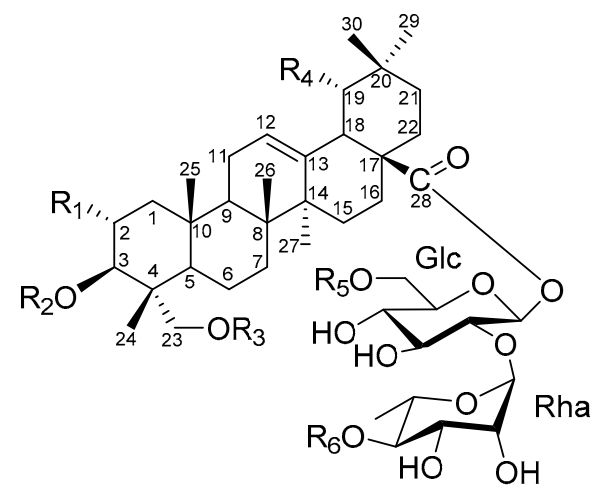

\begin{tabular}{ccccccc} 
& $\mathrm{R}_{1}$ & $\mathrm{R}_{2}$ & $\mathrm{R}_{3}$ & $\mathrm{R}_{4}$ & $\mathrm{R}_{5}$ & $\mathrm{R}_{6}$ \\
\hline $\mathbf{1}$ & $\mathrm{H}$ & $\mathrm{Xyl}$ & $\mathrm{Ac}$ & $\mathrm{H}$ & $\mathrm{Glc}$ & $\mathrm{Glc}$ \\
$\mathbf{2}$ & $\mathrm{H}$ & $\mathrm{Xyl}$ & $\mathrm{Ac}$ & $\mathrm{H}$ & $\mathrm{Ara}$ & $\mathrm{Glc}$ \\
$\mathbf{3}$ & $\mathrm{H}$ & $\mathrm{H}$ & $\mathrm{Ac}$ & $\mathrm{H}$ & Xyl & $\mathrm{Glc}$ \\
$\mathbf{4}$ & $\mathrm{H}$ & $\mathrm{Xyl}$ & $\mathrm{H}$ & $\mathrm{H}$ & Xyl & $\mathrm{Glc}$ \\
$\mathbf{5}$ & $\mathrm{H}$ & $\mathrm{Xyl}$ & $\mathrm{H}$ & $\mathrm{H}$ & Xyl & $\mathrm{H}$ \\
$\mathbf{6}$ & $\mathrm{OH}$ & $\mathrm{H}$ & $\mathrm{H}$ & $\mathrm{OH}$ & $\mathrm{H}$ & $\mathrm{H}$
\end{tabular}

Figure 1. Chemical structure of compounds 1-7 from Elsholtzia bodinieri.

Compound 1 was obtained as a white amorphous powder. Its molecular formula was determined as $\mathrm{C}_{61} \mathrm{H}_{98} \mathrm{O}_{28}$, according to the $[\mathrm{M}-\mathrm{H}]^{-}$peak at $m / z 1277.6148$ in the negative HR-ESI-MS, indicating 13 degrees of unsaturation. It exhibited a UV maximum at $204 \mathrm{~nm}$. The IR spectrum showed the presence of hydroxyl $\left(3441 \mathrm{~cm}^{-1}\right)$, carbonyl $\left(1722 \mathrm{~cm}^{-1}\right)$, and olefinic $\left(1635 \mathrm{~cm}^{-1}\right)$ groups. NMR data analysis indicated that 1 was a saponin containing a triterpene sapogenin and five monosaccharides.

In the ${ }^{1} \mathrm{H}$ and ${ }^{13} \mathrm{C}$ NMR spectra of aglycone moiety, 6 tertiary methyl groups $\left[\delta_{\mathrm{H}} 0.81\right.$, $0.83,0.84,0.88,1.07$, and 1.25 (each, $3 \mathrm{H}, \mathrm{s}$ ); $\delta_{\mathrm{C}} 12.9,15.9,17.5,23.6,25.5$, and 30.6, (each, q)], 11 methylenes containing an oxygenated one $\left[\delta_{\mathrm{H}} 4.03\left(1 \mathrm{H}, \mathrm{m}, \mathrm{H}_{\mathrm{a}}-23\right), 4.12(1 \mathrm{H}, \mathrm{m}\right.$, $\left.\mathrm{H}_{\mathrm{b}}-23 ; \delta_{\mathrm{C}} 64.7(\mathrm{t}, \mathrm{C}-23)\right], 5$ methines including an oxygenated one $\left[\delta_{\mathrm{H}} 3.73(1 \mathrm{H}, \mathrm{br} . \mathrm{s}, \mathrm{H}-3)\right.$; $\left.\delta_{\mathrm{C}} 81.9(\mathrm{~d}, \mathrm{C}-23)\right]$ and 1 unsaturated one $\left[\delta_{\mathrm{H}} 5.27(1 \mathrm{H}, \mathrm{m}, \mathrm{H}-12) ; \delta_{\mathrm{C}} 123.2(\mathrm{~d}, \mathrm{C}-12)\right]$, as well as 8 quaternary carbons (including a carbonyl carbon $\left(\delta_{\mathrm{C}} 176.4, \mathrm{C}-28\right)$ and a unsaturated one $\left.\left(\delta_{\mathrm{C}} 144.0, \mathrm{C}-13\right)\right)$ were observed (Tables 1 and 2$)$. This information suggested that the aglycone moiety of compound 1 was $3 \beta, 23$-dihydroxyolean-12-en-28-oic acid [12]. Except for the signals for the aglycone, the remaining 31 signals were assigned as five sugar moieties and an acetoxy group, due to signals of $\delta_{\mathrm{C}} 172.7$ and 20.9. Moreover, comparison of its ${ }^{1} \mathrm{H}$ and ${ }^{13} \mathrm{C}$ NMR spectroscopic data with those of Bodinioside $\mathrm{H}$ [4], suggested that they had same 3-hydroxy-23-acetoxy-olean-12-en-28-oic acid as the aglycone, but differed in the sugar moiety.

Table 1. H NMR data of compounds 1-7 in Pyridine- $d_{5}\left(600 \mathrm{MHz}, \delta_{\mathrm{H}}\right.$ in ppm, $J$ in $\left.\mathrm{Hz}\right)$

\begin{tabular}{|c|c|c|c|c|c|c|c|}
\hline Position & 1 & 2 & 3 & 4 & 5 & 6 & 7 \\
\hline 1 & $1.57(\mathrm{~m}) ; 0.96(\mathrm{~m})$ & $1.66(\mathrm{~m}) ; 0.98(\mathrm{~m})$ & $1.56(\mathrm{~m}) ; 0.82(\mathrm{~m})$ & $1.49(\mathrm{~m}) ; 1.15(\mathrm{~m})$ & $1.51(\mathrm{~m}) ; 1.03(\mathrm{~m})$ & $1.66(\mathrm{~m}) ; 1.17(\mathrm{~m})$ & $1.66(\mathrm{~m}) ; 1.23(\mathrm{~m})$ \\
\hline 2 & $1.81(\mathrm{~m}) ; 1.71(\mathrm{~m})$ & $1.92(\mathrm{~m}) ; 1.76(\mathrm{~m})$ & $1.89(\mathrm{~m}) ; 1.71(\mathrm{~m})$ & $2.20(\mathrm{~m}) ; 2.05(\mathrm{~m})$ & $2.20(\mathrm{~m}) ; 2.01(\mathrm{~m})$ & $4.24(\mathrm{~m})$ & $3.15(\mathrm{~m})$ \\
\hline 3 & $3.73(\mathrm{~m})$ & $3.59(\mathrm{~m})$ & $3.90(\mathrm{~m})$ & $4.31(\mathrm{~m})$ & $4.32(\mathrm{~m})$ & $3.41(\mathrm{~m})$ & $3.63(\mathrm{~m})$ \\
\hline 5 & $1.52(\mathrm{~m})$ & $1.74(\mathrm{~m})$ & $1.54(\mathrm{~m})$ & $1.69(\mathrm{~m})$ & $1.63(\mathrm{~m})$ & $1.02(\mathrm{~m})$ & $1.80(\mathrm{~m})$ \\
\hline 6 & $1.64(\mathrm{~m}) ; 1.35(\mathrm{~m})$ & $1.62(\mathrm{~m}) ; 1.40(\mathrm{~m})$ & $1.73(\mathrm{~m}) ; 1.33(\mathrm{~m})$ & $1.66(\mathrm{~m}) ; 1.37(\mathrm{~m})$ & $1.65(\mathrm{~m}) ; 1.31(\mathrm{~m})$ & $1.78(\mathrm{~m}) ; 1.54(\mathrm{~m})$ & $1.68(\mathrm{~m}) ; 1.44(\mathrm{~m})$ \\
\hline 7 & $1.82(\mathrm{~m}) ; 1.54(\mathrm{~m})$ & $1.71(\mathrm{~m}) ; 1.55(\mathrm{~m})$ & $1.93(\mathrm{~m}) ; 1.57(\mathrm{~m})$ & $1.87(\mathrm{~m}) ; 1.52(\mathrm{~m})$ & $1.84(\mathrm{~m}) ; 1.50(\mathrm{~m})$ & $1.67(\mathrm{~m}) ; 1.35(\mathrm{~m})$ & $1.73(\mathrm{~m}) ; 1.36(\mathrm{~m})$ \\
\hline 9 & $1.66(\mathrm{~m})$ & $1.68(\mathrm{~m})$ & $1.76(\mathrm{~m})$ & $1.70(\mathrm{~m})$ & $1.70(\mathrm{~m})$ & $1.80(\mathrm{~m})$ & $1.61(\mathrm{~m})$ \\
\hline 11 & $1.93(\mathrm{~m}) ; 1.87(\mathrm{~m})$ & $1.93(\mathrm{~m}) ; 1.82(\mathrm{~m})$ & $1.92(\mathrm{~m}) ; 1.86(\mathrm{~m})$ & $1.97(\mathrm{~m}) ; 1.82(\mathrm{~m})$ & $1.96(\mathrm{~m}) ; 1.84(\mathrm{~m})$ & 2.02(m); $1.92(\mathrm{~m})$ & $1.98(\mathrm{~m}) ; 1.62(\mathrm{~m})$ \\
\hline 12 & 5.27 (br. s) & 5.42 (br. s) & 5.44 (br. s) & 5.41 (br. s) & 5.40 (br. s) & 5.52 (br. s) & 5.55 (br. s) \\
\hline 15 & $1.67(\mathrm{~m}) ; 1.17(\mathrm{~m})$ & $1.57(\mathrm{~m}) ; 1.20(\mathrm{~m})$ & $1.78(\mathrm{~m}) ; 1.21(\mathrm{~m})$ & $2.06(\mathrm{~m}) ; 1.56(\mathrm{~m})$ & $2.04(\mathrm{~m}) ; 1.55(\mathrm{~m})$ & $2.05(\mathrm{~m}) ; 1.69(\mathrm{~m})$ & $2.03(\mathrm{~m}) ; 1.36(\mathrm{~m})$ \\
\hline 16 & $2.03(\mathrm{~m}) ; 1.72(\mathrm{~m})$ & $2.03(\mathrm{~m}) ; 1.68(\mathrm{~m})$ & $2.01(\mathrm{~m}) ; 1.56(\mathrm{~m})$ & $2.20(\mathrm{~m}) ; 2.18(\mathrm{~m})$ & $2.12(\mathrm{~m}) ; 2.07(\mathrm{~m})$ & $2.28(\mathrm{~m}) ; 2.10(\mathrm{~m})$ & $2.24(\mathrm{~m}) ; 2.12(\mathrm{~m})$ \\
\hline 18 & $3.12(\mathrm{dd}, 13.6,3.8)$ & $3.14(\mathrm{~d}, 13.5)$ & $3.17(\mathrm{~d}, 13.5)$ & $3.13(\mathrm{~d}, 13.7)$ & $3.11(\mathrm{dd}, 13.5,4.0)$ & $2.88(\mathrm{dd}, 13.5,3.4)$ & $2.87(\mathrm{~s})$ \\
\hline 19 & $1.70(\mathrm{~m}) ; 1.17(\mathrm{~m})$ & $2.08(\mathrm{~m}) ; 1.18(\mathrm{~m})$ & $1.89(\mathrm{~m}) ; 1.12(\mathrm{~m})$ & $1.74(\mathrm{~m}) ; 1.15(\mathrm{~m})$ & $1.76(\mathrm{~m}) ; 1.12(\mathrm{~m})$ & & \\
\hline 21 & $1.28(\mathrm{~m}) ; 1.19(\mathrm{~m})$ & $2.20(\mathrm{~m}) ; 1.14(\mathrm{~m})$ & $1.29(\mathrm{~m}) ; 1.01(\mathrm{~m})$ & $1.42(\mathrm{~m}) ; 1.04(\mathrm{~m})$ & $1.30(\mathrm{~m}) ; 0.94(\mathrm{~m})$ & $2.04(\mathrm{~m}) ; 1.72(\mathrm{~m})$ & $2.04(\mathrm{~m}) ; 1.78(\mathrm{~m})$ \\
\hline 22 & $1.68(\mathrm{~m}) ; 1.32(\mathrm{~m})$ & $1.39(\mathrm{~m}) ; 1.00(\mathrm{~m})$ & $1.75(\mathrm{~m}) ; 1.35(\mathrm{~m})$ & $1.68(\mathrm{~m}) ; 1.35(\mathrm{~m})$ & $1.64(\mathrm{~m}) ; 1.08(\mathrm{~m})$ & $\begin{array}{l}2.08(\mathrm{~m}) ; 1.78 \\
\quad \text { (overlap) }\end{array}$ & $2.29(\mathrm{~m}) ; 1.42(\mathrm{~m})$ \\
\hline
\end{tabular}


Table 1. Cont

\begin{tabular}{|c|c|c|c|c|c|c|c|}
\hline Position & 1 & 2 & 3 & 4 & 5 & 6 & 7 \\
\hline 23 & $4.12(\mathrm{~m}) ; 4.03(\mathrm{~m})$ & $4.45(\mathrm{~m}) ; 4.03(\mathrm{~m})$ & $4.30(\mathrm{~m}) ; 4.10(\mathrm{~m})$ & $4.32(\mathrm{~m}) ; 3.63(\mathrm{~m})$ & $4.33(\mathrm{~m}) ; 3.61(\mathrm{~m})$ & $4.36(\mathrm{~m}) ; 3.04(\mathrm{~m})$ & $4.33(\mathrm{~m}) ; 4.02(\mathrm{~m})$ \\
\hline 24 & $0.83(\mathrm{~s})$ & $0.84(\mathrm{~s})$ & $0.88(\mathrm{~s})$ & $0.90(\mathrm{~s})$ & $0.87(\mathrm{~s})$ & $1.04(\mathrm{~s})$ & $1.13(\mathrm{~s})$ \\
\hline 25 & $1.07(\mathrm{~s})$ & $1.09(\mathrm{~s})$ & $1.14(\mathrm{~s})$ & $1.20(\mathrm{~s})$ & $1.09(\mathrm{~s})$ & $1.09(\mathrm{~s})$ & $1.39(\mathrm{~s})$ \\
\hline 26 & $0.81(\mathrm{~s})$ & $0.83(\mathrm{~s})$ & $0.84(\mathrm{~s})$ & $0.82(\mathrm{~s})$ & $0.82(\mathrm{~s})$ & $0.84(\mathrm{~s})$ & $1.03(\mathrm{~s})$ \\
\hline 27 & $0.84(\mathrm{~s})$ & $0.89(\mathrm{~s})$ & $0.93(\mathrm{~s})$ & $0.99(\mathrm{~s})$ & $0.90(\mathrm{~s})$ & $1.15(\mathrm{~s})$ & $1.19(\mathrm{~s})$ \\
\hline 29 & $1.25(\mathrm{~s})$ & $1.26(\mathrm{~s})$ & $0.97(\mathrm{~s})$ & $1.23(\mathrm{~s})$ & $0.97(\mathrm{~s})$ & $1.54(\mathrm{~s})$ & $1.62(\mathrm{~s})$ \\
\hline 30 & $0.88(\mathrm{~s})$ & $0.91(\mathrm{~s})$ & $1.26(\mathrm{~s})$ & $1.48(\mathrm{~s})$ & $1.16(\mathrm{~s})$ & $1.11(\mathrm{~s})$ & $1.06(\mathrm{~s})$ \\
\hline $23-\mathrm{A}_{\mathrm{C}} \mathrm{O}$ & $2.15(\mathrm{~s})$ & $2.14(\mathrm{~s})$ & $2.08(\mathrm{~s})$ & & & & \\
\hline \multicolumn{8}{|l|}{ 3-Xyl } \\
\hline 1 & $4.97(\mathrm{~d}, 7.8)$ & $4.86(\mathrm{~d}, 7.8)$ & & $5.02(\mathrm{~d}, 7.4)$ & $5.01(\mathrm{~d}, 7.8)$ & & \\
\hline 2 & $4.22(\mathrm{~m})$ & $4.20(\mathrm{~m})$ & & $4.08(\mathrm{~m})$ & $4.08(\mathrm{~m})$ & & \\
\hline 3 & $4.06(\mathrm{~m})$ & $4.14(\mathrm{~m})$ & & $4.14(\mathrm{~m})$ & $3.99(t, 8.3)$ & & \\
\hline 4 & $4.12(\mathrm{~m})$ & $4.35(\mathrm{~m})$ & & $4.21(\mathrm{~m})$ & $4.13(\mathrm{~m})$ & & \\
\hline 5 & $3.75(\mathrm{~m}) ; 3.73(\mathrm{~m})$ & $4.42(\mathrm{~m}) ; 3.74(\mathrm{~m})$ & & $4.32(\mathrm{~m}) ; 3.63(\mathrm{~m})$ & $3.64(\mathrm{~m}) ; 3.60(\mathrm{~m})$ & & \\
\hline \multicolumn{8}{|l|}{$28-O-$} \\
\hline \multicolumn{8}{|l|}{$\begin{array}{l}\text { sugar } \\
\text { Glc }\end{array}$} \\
\hline 1 & $6.09(\mathrm{~d}, 7.8)$ & $6.14(\mathrm{~d}, 7.8)$ & $6.15(\mathrm{~d}, 7.8)$ & $6.11(\mathrm{~d}, 7.9)$ & $6.11(\mathrm{~d}, 8.0)$ & $6.23(\mathrm{~d}, 8.1)$ & $6.16(\mathrm{~d}, 7.9)$ \\
\hline 2 & $4.27(\mathrm{~m})$ & $4.58(\mathrm{~m})$ & $3.97(t, 8.1)$ & $4.28(\mathrm{~m})$ & $4.36(t, 8.3)$ & $4.01(\mathrm{~m})$ & $4.15(\mathrm{~m})$ \\
\hline 3 & $4.25(\mathrm{~m})$ & $4.07(\mathrm{~m})$ & $4.05(\mathrm{~m})$ & $4.10(\mathrm{~m})$ & $4.04(t, 8.8)$ & $4.24(\mathrm{~m})$ & $4.61(\mathrm{~d}, 9.8)$ \\
\hline 4 & $4.30(\mathrm{~m})$ & $4.54(\mathrm{~m})$ & $3.89(\mathrm{~m})$ & $4.23(\mathrm{~m})$ & $4.23(\mathrm{~m})$ & $4.17(\mathrm{~d}, 9.5)$ & $4.02(\mathrm{~d}, 9.8)$ \\
\hline 5 & $4.14(\mathrm{~m})$ & $4.16(\mathrm{~m})$ & $3.78(\mathrm{~m})$ & $4.18(\mathrm{~m})$ & $4.30(\mathrm{~m})$ & $4.60(\mathrm{~m})$ & $4.32(\mathrm{~m})$ \\
\hline 6 & $4.70(\mathrm{~m}) ; 4.28(\mathrm{~m})$ & $4.81(\mathrm{~m}) ; 4.65(\mathrm{~m})$ & $\begin{array}{l}4.64(\mathrm{dd}, 11.4, \\
2.2) ; 4.45(\mathrm{~m})\end{array}$ & $\begin{array}{c}4.43(\mathrm{~d}, 10.2) \\
4.38(\mathrm{~m})\end{array}$ & $\begin{array}{l}4.62(\mathrm{~d}, 11.0) \\
4.35(\mathrm{~m})\end{array}$ & $\begin{array}{l}3.64(\mathrm{dd}, 11.5,5.2) \\
3.52(\mathrm{dd}, 11.5,2.0)\end{array}$ & $\begin{array}{c}4.46(\mathrm{dd}, 10.8 \\
4.2) ; 4.24(\mathrm{~d}, 10.8)\end{array}$ \\
\hline \multicolumn{8}{|c|}{$0.02(40,11.0,2.0)$} \\
\hline 1 & 6.45 (br. s) & 6.47 (br. s) & 6.45 (br. s) & 6.38 (br. s) & 6.51 (br. s) & 6.60 (br. s) & 6.58 (br. s) \\
\hline 2 & $4.81(\mathrm{~m})$ & 4.81 (overlap) & 4.81 (br. s) & 4.81 (br. s) & 4.76 (br. s) & 4.83 (br. s) & 4.81 (br. s) \\
\hline 3 & 4.64 (overlap) & $4.77(\mathrm{~m})$ & $4.50(\mathrm{~m})$ & $4.92(\mathrm{~m})$ & $4.52(\mathrm{~m})$ & $4.52(\mathrm{~m})$ & $4.02(\mathrm{~m})$ \\
\hline 4 & $4.48(\mathrm{~m})$ & $4.42(\mathrm{~m})$ & $4.42(\mathrm{~m})$ & $4.45(\mathrm{~m})$ & $4.28(\mathrm{~m})$ & $4.40(\mathrm{~m})$ & $4.15(\mathrm{~d}, 9.8)$ \\
\hline 5 & $4.39(\mathrm{~m})$ & $4.37(\mathrm{~m})$ & $4.39(\mathrm{~m})$ & $4.37(\mathrm{~m})$ & $4.16(\mathrm{~m})$ & $4.14(\mathrm{~m})$ & $4.61(\mathrm{~m})$ \\
\hline 6 & $1.81(\mathrm{~d}, 6.1)$ & $1.82(\mathrm{~d}, 6.0)$ & $1.82(\mathrm{~d}, 6.0)$ & $1.80(\mathrm{~d}, 6.0)$ & $1.73(\mathrm{~d}, 6.0)$ & $1.78(\mathrm{~d}, 6.0)$ & $1.75(\mathrm{~d}, 6.0)$ \\
\hline \multicolumn{8}{|c|}{$\mathrm{Glc}^{\prime} / \mathrm{Ara} / \mathrm{Xyl}$} \\
\hline 1 & $4.86(\mathrm{~d}, 7.8)$ & 6.62 (br. s) & $5.13(\mathrm{~d}, 7.8)$ & $4.85(\mathrm{~d}, 7.4)$ & $4.85(\mathrm{~d}, 7.4)$ & & \\
\hline 2 & $4.02(\mathrm{~m})$ & $4.06(\mathrm{~m})$ & $4.06(\mathrm{~m})$ & $4.26(\mathrm{~m})$ & $3.94(t, 8.0)$ & & \\
\hline 3 & $4.08(\mathrm{~m})$ & $4.12(\mathrm{~m})$ & $4.71(\mathrm{dd}, 9.5,3.2)$ & $4.17(\mathrm{~m})$ & $4.25(\mathrm{~m})$ & & \\
\hline 4 & $4.17(\mathrm{~m})$ & $4.28(\mathrm{~m})$ & $4.28(\mathrm{~m})$ & $4.02(\mathrm{~m})$ & $4.02(\mathrm{~m})$ & & \\
\hline 5 & $3.87(\mathrm{~m})$ & $4.45(\mathrm{~m}) ; 3.73(\mathrm{~m})$ & $3.87(\mathrm{~m}) ; 3.76(\mathrm{~m})$ & $3.72(\mathrm{~m}) ; 3.70(\mathrm{~m})$ & $3.65(\mathrm{~m}) ; 3.61(\mathrm{~m})$ & & \\
\hline 6 & $\begin{array}{c}4.57(\mathrm{~d}, 11.2) \\
4.30(\mathrm{~m})\end{array}$ & & & & & & \\
\hline \multicolumn{8}{|l|}{ Glc" / Glc' } \\
\hline 1 & $5.16(\mathrm{~d}, 7.8)$ & $5.12(\mathrm{~d}, 7.8)$ & $4.89(\mathrm{~d}, 7.8)$ & $5.15(\mathrm{~d}, 7.6)$ & & & \\
\hline 2 & $4.45(\mathrm{~m})$ & $4.57(\mathrm{~m})$ & $4.09(\mathrm{~m})$ & $3.97(\mathrm{~m})$ & & & \\
\hline 3 & $4.38(\mathrm{~m})$ & $4.02(\mathrm{~m})$ & $4.18(\mathrm{~m})$ & $4.12(\mathrm{~m})$ & & & \\
\hline 4 & $4.30(\mathrm{~m})$ & $4.46(\mathrm{~m})$ & $3.65(t, 9.5)$ & $4.21(\mathrm{~m})$ & & & \\
\hline 5 & $3.94(\mathrm{~m})$ & $3.98(\mathrm{~m})$ & $4.30(\mathrm{~m})$ & $3.78(\mathrm{~m})$ & & & \\
\hline 6 & $\begin{array}{c}4.65(\mathrm{~d}, 11.2) \\
4.28(\mathrm{~m})\end{array}$ & $\begin{array}{c}4.81(\mathrm{~d}, 11.0) ; 4.67 \\
(\mathrm{~m})\end{array}$ & $\begin{array}{l}4.64(\mathrm{dd}, 11.4, \\
2.2) ; 4.45(\mathrm{~m})\end{array}$ & $\begin{array}{c}4.42(\mathrm{~d}, 11.2) ; 4.33 \\
(\mathrm{~m})\end{array}$ & & & \\
\hline
\end{tabular}

Table 2. C NMR data of compounds $1-7$ in pyridine- $d_{5}\left(150 \mathrm{MHz}, \delta_{\mathrm{C}}\right.$ in ppm).

\begin{tabular}{|c|c|c|c|c|c|c|c|}
\hline Position & 1 & 2 & 3 & 4 & 5 & 6 & 7 \\
\hline 1 & 39.8 & 38.5 & 38.6 & 39.2 & 38.6 & 47.7 & 47.9 \\
\hline 2 & 26.0 & 26.4 & 26.8 & 26.2 & 26.1 & 68.9 & 68.8 \\
\hline 3 & 81.9 & 81.9 & 78.3 & 82.1 & 81.9 & 78.5 & 79.9 \\
\hline 4 & 42.4 & 42.1 & 42.2 & 43.6 & 43.3 & 43.7 & 43.5 \\
\hline 5 & 48.3 & 48.3 & 48.3 & 48.0 & 47.0 & 48.2 & 48.0 \\
\hline 6 & 18.5 & 18.4 & 18.6 & 18.4 & 18.1 & 18.9 & 18.6 \\
\hline 7 & 33.7 & 32.2 & 32.3 & 33.2 & 32.2 & 33.2 & 33.3 \\
\hline 8 & 41.8 & 39.8 & 41.8 & 40.1 & 39.8 & 40.3 & 40.5 \\
\hline 9 & 48.2 & 48.3 & 48.3 & 48.3 & 47.6 & 48.5 & 41.7 \\
\hline 10 & 36.7 & 36.8 & 37.1 & 37.1 & 36.8 & 38.6 & 38.3 \\
\hline 11 & 23.6 & 23.7 & 23.2 & 25.1 & 23.7 & 24.6 & 24.2 \\
\hline 12 & 123.2 & 123.3 & 123.2 & 123.1 & 122.6 & 123.5 & 128.2 \\
\hline 13 & 144.0 & 144.0 & 143.9 & 144.2 & 143.9 & 144.5 & 139.2 \\
\hline 14 & 42.1 & 41.8 & 42.3 & 42.3 & 41.8 & 42.5 & 42.2 \\
\hline 15 & 30.6 & 28.8 & 28.7 & 28.4 & 28.5 & 28.8 & 29.4 \\
\hline 16 & 23.1 & 23.3 & 23.8 & 23.7 & 23.3 & 24.4 & 26.1 \\
\hline 17 & 47.1 & 47.1 & 48.6 & 47.5 & 48.1 & 46.7 & 48.0 \\
\hline 18 & 42.1 & 42.4 & 42.1 & 42.3 & 42.1 & 45.0 & 54.6 \\
\hline 19 & 46.2 & 46.6 & 46.3 & 47.0 & 46.3 & 81.3 & 72.5 \\
\hline
\end{tabular}


Table 2. Cont.

\begin{tabular}{|c|c|c|c|c|c|c|c|}
\hline Position & 1 & 2 & 3 & 4 & 5 & 6 & 7 \\
\hline 20 & 30.3 & 30.6 & 30.6 & 30.8 & 30.6 & 29.5 & 42.2 \\
\hline 21 & 32.9 & 34.2 & 34.4 & 36.7 & 33.8 & 35.4 & 26.7 \\
\hline 22 & 32.1 & 33.8 & 33.1 & 32.9 & 32.8 & 32.7 & 37.4 \\
\hline 23 & 64.7 & 65.6 & 65.2 & 64.3 & 64.3 & 66.7 & 66.6 \\
\hline 24 & 12.9 & 13.0 & 12.7 & 13.7 & 13.5 & 14.3 & 14.2 \\
\hline 25 & 15.9 & 15.9 & 16.0 & 16.4 & 16.1 & 17.7 & 17.5 \\
\hline 26 & 17.5 & 17.4 & 17.5 & 17.7 & 17.4 & 17.5 & 17.4 \\
\hline 27 & 25.5 & 25.6 & 25.7 & 26.1 & 25.7 & 28.0 & 24.1 \\
\hline 28 & 176.4 & 176.5 & 176.5 & 176.7 & 176.4 & 177.2 & 176.8 \\
\hline 29 & 30.6 & 33.0 & 33.0 & 33.2 & 33.0 & 33.2 & 26.9 \\
\hline 30 & 23.6 & 23.7 & 23.7 & 23.7 & 23.7 & 24.7 & 16.6 \\
\hline \multirow[t]{2}{*}{$23-\mathrm{A}_{\mathrm{C}} \mathrm{O}$} & 171.2 & 171.1 & 171.1 & & & & \\
\hline & 20.7 & 20.7 & 20.7 & & & & \\
\hline \multicolumn{8}{|l|}{$3-X y 1$} \\
\hline 1 & 107.1 & 107.2 & & 107.2 & 106.8 & & \\
\hline 2 & 75.3 & 75.3 & & 75.7 & 75.4 & & \\
\hline 3 & 78.4 & 78.4 & & 78.1 & 77.8 & & \\
\hline 4 & 71.5 & 71.1 & & 71.3 & 71.0 & & \\
\hline 5 & 67.1 & 67.1 & & 67.2 & 66.8 & & \\
\hline \multicolumn{8}{|l|}{ 28-O-sugar } \\
\hline \multicolumn{8}{|l|}{ Glc } \\
\hline 1 & 94.5 & 94.4 & 94.5 & 94.7 & 94.6 & 95.1 & 94.8 \\
\hline 2 & 76.3 & 76.3 & 76.3 & 77.6 & 76.2 & 75.7 & 75.1 \\
\hline 3 & 78.5 & 79.2 & 78.3 & 78.5 & 78.4 & 79.8 & 78.9 \\
\hline 4 & 71.4 & 71.5 & 71.1 & 71.1 & 71.8 & 71.4 & 72.5 \\
\hline 5 & 77.6 & 77.3 & 77.4 & 77.6 & 77.9 & 79.1 & 78.3 \\
\hline 6 & 68.2 & 68.3 & 68.3 & 68.6 & 69.7 & 62.1 & 62.2 \\
\hline \multicolumn{8}{|l|}{ Rha } \\
\hline 1 & 101.0 & 101.2 & 101.2 & 101.5 & 101.3 & 101.4 & 101.3 \\
\hline 2 & 71.3 & 71.6 & 71.6 & 71.3 & 70.9 & 72.2 & 71.4 \\
\hline 3 & 72.4 & 72.4 & 72.3 & 72.1 & 72.3 & 72.4 & 72.2 \\
\hline 4 & 85.7 & 85.7 & 85.7 & 85.6 & 74.6 & 73.8 & 73.7 \\
\hline 5 & 70.9 & 71.5 & 70.9 & 71.1 & 71.8 & 69.7 & 69.6 \\
\hline 6 & 18.6 & 18.5 & 18.7 & 18.8 & 18.6 & 18.6 & 18.6 \\
\hline \multicolumn{8}{|c|}{ Glc' / Ara / Xyl } \\
\hline 1 & 105.3 & 109.7 & 105.5 & 105.7 & 105.4 & & \\
\hline 2 & 75.3 & 81.9 & 74.7 & 74.9 & 75.5 & & \\
\hline 3 & 78.5 & 76.3 & 77.9 & 78.7 & 77.8 & & \\
\hline 4 & 71.3 & 86.0 & 72.4 & 71.8 & 72.0 & & \\
\hline 5 & 78.2 & 67.2 & 66.9 & 67.2 & 66.8 & & \\
\hline 6 & 62.7 & & & & & & \\
\hline \multicolumn{8}{|l|}{$\mathrm{Glc}^{\prime \prime} / \mathrm{Glc}^{\prime}$} \\
\hline 1 & 107.2 & 107.0 & 107.1 & 106.7 & & & \\
\hline 2 & 76.3 & 76.3 & 75.8 & 75.5 & & & \\
\hline 3 & 79.2 & 79.2 & 79.3 & 78.8 & & & \\
\hline 4 & 71.0 & 71.5 & 71.6 & 71.8 & & & \\
\hline 5 & 78.5 & 78.2 & 77.4 & 78.7 & & & \\
\hline 6 & 62.5 & 62.7 & 62.8 & 62.9 & & & \\
\hline
\end{tabular}

The ${ }^{1} \mathrm{H}$ NMR spectrum showed five signals for anomeric protons at $\delta_{\mathrm{H}} 6.45(1 \mathrm{H}$, br. s), $6.09(1 \mathrm{H}, \mathrm{d}, J=7.8 \mathrm{~Hz}), 5.16(1 \mathrm{H}, \mathrm{d}, J=7.8 \mathrm{~Hz}), 4.97(1 \mathrm{H}, \mathrm{d}, J=7.8 \mathrm{~Hz})$ and $4.86(1 \mathrm{H}, \mathrm{d}, J=7.8 \mathrm{~Hz})$, which correlated with anomeric carbon signals at $\delta_{\mathrm{C}} 101.0,94.5$, 107.2, 107.1 and 105.3 in the HSQC spectrum, respectively, suggesting the presence of five sugar moieties. Acid hydrolysis of 1 with $1 \mathrm{M} \mathrm{HCl}$ produced L-rhamnose (Rha), D-glucose (Glc), and D-xylose (Xyl) as sugar residues by GC chromatography with the corresponding trimethylsilylated L-cysteine derivatives. Since NMR signals of five sugar units have undesirable overlapped effects, the HMQC-TOCSY experiment was successfully 
used to distinguish and assign the ${ }^{1} \mathrm{H}$ and ${ }^{13} \mathrm{C}$ NMR signals of each sugar moiety. The correlations from the anomeric proton signal at $\delta_{\mathrm{H}} 4.97$ to three carbon signals at $\delta_{\mathrm{C}}$ $75.3,78.4$, and 67.1, as well as from three proton signals at $\delta_{\mathrm{H}} 4.22,4.06$, and 3.75 to the anomeric carbon, suggested the presence of D-xylopyranose. In a similar way, the ${ }^{1} \mathrm{H}$ and ${ }^{13} \mathrm{C}$ NMR signals for D-glucopyranosyl and L-rhamnopyranosyl were assigned. In addition, the $J \mathrm{H} 1, \mathrm{H} 2$ coupling constants of four anomeric proton signals at $\delta_{\mathrm{H}} 6.09(1 \mathrm{H}$, $\mathrm{d}, J=7.8 \mathrm{~Hz}), 5.16(1 \mathrm{H}, \mathrm{d}, J=7.8 \mathrm{~Hz}), 4.97(1 \mathrm{H}, \mathrm{d}, J=7.8 \mathrm{~Hz})$ and $4.86(1 \mathrm{H}, \mathrm{d}, J=7.8 \mathrm{~Hz})$ suggested the $\beta$ anomeric configuration for the xylopyranosyl and glucopyranosyl units. The inspection of the anomeric proton $(6.45, \mathrm{br}$. s) deduced the $\alpha$ anomeric configuration for the L-rhamnopyranosyl unit [4].

The sequence of the glycoside chains connected to C-3 and C-28 was established by analysis of the HMBC correlations (Figure 2). The absence of any glycosidation shift for $\mathrm{Xyl}$ suggested that $\mathrm{Xyl}$ was the singlet sugar unit attached at C-3 of the aglycone, which was further confirmed by $\mathrm{HMBC}$ correlation of $\mathrm{H}_{\mathrm{Xyl}^{-}}-1\left(\delta_{\mathrm{H}} 4.97\right)$ of C-3. A series of $\mathrm{HMBC}$ correlations from $\mathrm{H}_{\mathrm{Glc}}-1\left(\delta_{\mathrm{H}} 6.09\right)$ to $\mathrm{C}-28$, from $\mathrm{H}_{\mathrm{Rha}}-1\left(\delta_{\mathrm{H}} 6.45\right)$ to $\mathrm{C}_{\mathrm{Glc}}-2$ ( $\delta \mathrm{c} 76.3)$, from $\mathrm{H}_{\mathrm{Glc}^{\prime}}-1\left(\delta_{\mathrm{H}} 4.86\right)$ to $\mathrm{C}_{\mathrm{Glc}^{-}}-6(\delta \mathrm{c} 68.2)$, and from $\mathrm{H}_{\mathrm{Glc}^{\prime \prime}}-1\left(\delta_{\mathrm{H}} 5.16\right)$ to $\mathrm{C}_{\mathrm{Rha}^{-}}-4$ $(\delta \mathrm{c} 85.7)$ enabled the sugar chain of $\mathrm{C}-28$ to be assigned as $\beta$-D-glucopyranosyl $(1 \rightarrow 6)$ [ $\beta$-D-glucopyranosyl-( $1 \rightarrow 4)$ - $\alpha$-L-rhamnopyranosyl $(1 \rightarrow 2)]-\beta$-D-glucopyranoside. Thus, the structure of compound 1 was elucidated to be 3-O- $\beta$-D-xylopyranosyl-23-acetyloxyolean-12-en-28-oic acid 28-O- $\beta$-D-glucopyranosyl $(1 \rightarrow 6)$-[ $\beta$-D-glucopyranosyl-( $(1 \rightarrow 4)-\alpha$-Lrhamnopyranosyl $(1 \rightarrow 2)]-\beta$-D-glucopyranoside, a new oleanane triterpenoid saponin, named Bodinioside S.

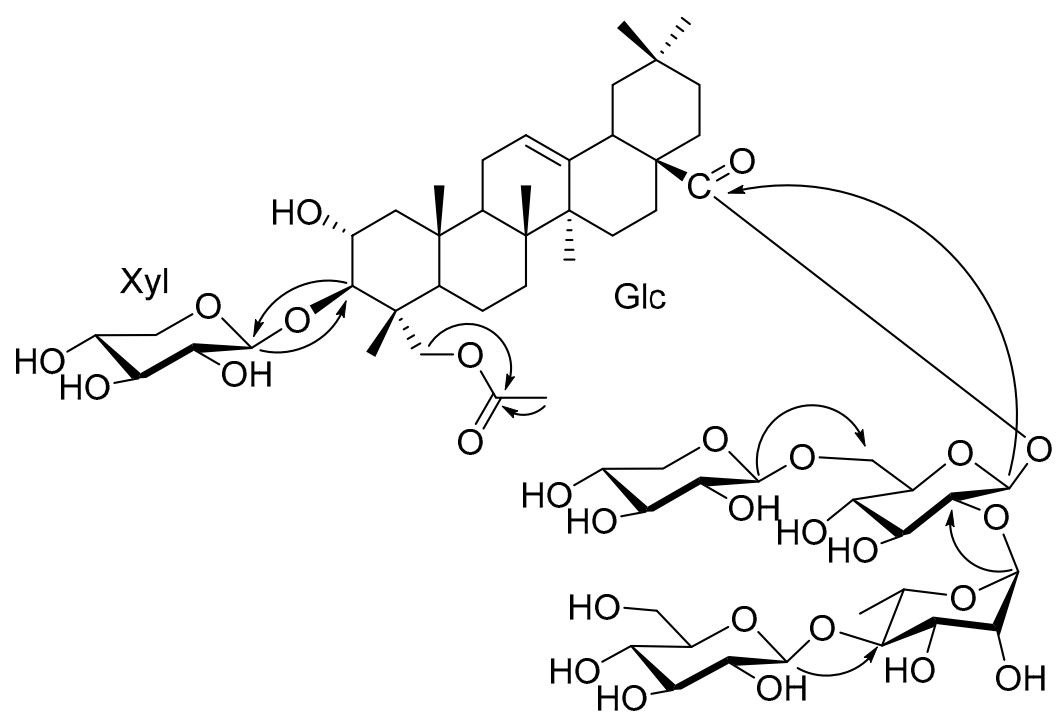

1

Figure 2. Key HMBC correlation of compound $\mathbf{1 .}$

Compound 2 was obtained as white amorphous powder. Its positive HR-ESI-MS spectrum indicated the molecular formula to be $\mathrm{C}_{60} \mathrm{H}_{96} \mathrm{O}_{27}$ by the observation quasi-molecular ion peak $[\mathrm{M}-\mathrm{H}]^{-}$at $m / z 1247.6070$ and with the help of the NMR spectroscopic date, indicating 13 degrees of unsaturation. Detailed comparison of the ${ }^{1} \mathrm{H}$ and ${ }^{13} \mathrm{C}$ NMR spectral data (Tables 1 and 2) of $\mathbf{2}$ with those of $\mathbf{1}$ revealed that they were highly structural similar, except for the replacement of signals for the Glc' at C-28 in $\mathbf{1}$ by the Ara in $\mathbf{2}$. Acid hydrolysis of $\mathbf{2}$ yielded L-rhamnose, D-glucose, L-arabinose and D-xylose as sugar residues as determined by GC analysis. In the ${ }^{1} \mathrm{H}$ NMR spectrum of 2 , five anomeric $\mathrm{H}$-atom at $\delta_{\mathrm{H}} 6.47$ (br. s), $6.14(\mathrm{~d}, J=7.8 \mathrm{~Hz}), 5.62$ (br. s), $5.12(\mathrm{~d}, J=7.8 \mathrm{~Hz})$ and $4.86(\mathrm{~d}, J=7.5 \mathrm{~Hz})$ correlated with anomeric carbon signals at $\delta_{\mathrm{C}} 101.2,94.4,109.7,107.0$, and 107.2 in the HSQC spectrum, respectively, suggesting the presence of five sugar residues: one rhamnopyranosyl (Rha), one arabinopyranosyl (Ara), one xylopyranosyl (Xyl) and two glucopyranosyl (Glc and 
Glc') units. The Xyl unit was still linked to $\mathrm{C}-3\left(\delta_{\mathrm{C}} 82.8\right)$ of the aglycone based on the HMBC correlation between $\mathrm{H}_{\mathrm{Xyl}}-1\left(\delta_{\mathrm{H}} 4.86\right)$ of $\mathrm{Xyl}$ and $\mathrm{C}-3(\delta \mathrm{c} 82.8)$. A series of HMBC correlations from $\mathrm{H}_{\mathrm{Glc}}-1\left(\delta_{\mathrm{H}} 6.14\right)$ to $\mathrm{C}-28$, from $\mathrm{H}_{\mathrm{Rha}}-1\left(\delta_{\mathrm{H}} 6.47\right)$ to $\mathrm{C}_{\mathrm{Glc}}-2(\delta \mathrm{c} 76.3)$, from $\mathrm{H}_{\mathrm{Ara}}-1\left(\delta_{\mathrm{H}} 5.62\right)$ to $\mathrm{C}_{\mathrm{Glc}^{-}}-6(\delta \mathrm{c} 68.3)$, and from $\mathrm{H}_{\mathrm{Glc}^{\prime}}-1\left(\delta_{\mathrm{H}} 5.12\right)$ to $\mathrm{C}_{\mathrm{Rha}}-4(\delta \mathrm{c} 85.7)$ enabled the sugar chain of $\mathrm{C}-28$ to be assigned as $\alpha$-L -arabinopyranosyl( $1 \rightarrow 6)$-[ $\beta$-D-glucopyranosyl$(1 \rightarrow 4)$ - $\alpha$-L-rhamnopyranosyl $(1 \rightarrow 2)]-\beta$-D-glucopyranoside. From the foregoing evidence, the structure of 1 was unequivocally determined to be 3-O- $\beta$-D-xylopyranosyl-23-acetyloxyolean-12-en-28-oic acid 28-O- $\alpha$-L-arabinopyranosyl( $1 \rightarrow 6)$-[ $\beta$-D-glucopyranosyl-( $1 \rightarrow 4)-\alpha$-Lrhamnopyranosyl-( $1 \rightarrow 2)]-\beta$-D-glucopyranoside, and named Bodinioside $\mathrm{T}$.

Compound 3 was isolated as white amorphous powder. The molecular formula was established as $\mathrm{C}_{55} \mathrm{H}_{88} \mathrm{O}_{23}$ by positive HR-ESI-MS $\left(\mathrm{m} / z\right.$ 1139.5614, $\left.[\mathrm{M}+\mathrm{Na}]^{+}\right)$and NMR spectral data, indicating 12 degrees of unsaturation. In the ${ }^{1} \mathrm{H}$ NMR spectrum of 3 , four anomeric $\mathrm{H}$-atom at $\delta_{\mathrm{H}} 6.45(\mathrm{br} . \mathrm{s}), 6.15(\mathrm{~d}, J=7.8 \mathrm{~Hz}), 5.13(\mathrm{~d}, J=7.8 \mathrm{~Hz})$ and $4.89(\mathrm{~d}$, $J=7.8 \mathrm{~Hz}$ ) correlated with anomeric carbon signals at $\delta_{\mathrm{C}} 101.2,94.5,105.5$ and 107.1 in the HSQC spectrum, respectively, suggesting the presence of four sugar residues: one rhamnopyranosyl (Rha), one xylopyranosyl (Xyl), and two glucopyranosyl (Glc and Glc') units. Detailed comparison of the ${ }^{1} \mathrm{H}$ and ${ }^{13} \mathrm{C}$ NMR spectra of 3 (Tables 1 and 2) with those of Bodinioside $\mathrm{H}$ [4] revealed that they were identical, except for the absence of signals for Xyl moiety on C-3. GC analysis after acid hydrolysis of $\mathbf{3}$ as the same manner with 1 gave D-glucose, D-xylose, and L-rhamnose in a ratio of 2:1:1. Moreover, a series of $\mathrm{HMBC}$ correlations from $\mathrm{H}_{\mathrm{Glc}}-1\left(\delta_{\mathrm{H}} 6.15\right)$ to $\mathrm{C}-28$, from $\mathrm{H}_{\mathrm{Rha}}-1\left(\delta_{\mathrm{H}} 6.45\right)$ to $\mathrm{C}_{\mathrm{Glc}}-2(\delta \mathrm{c}$ 76.3), from $\mathrm{H}_{\mathrm{Xyl}^{-1}}-1\left(\delta_{\mathrm{H}} 5.13\right)$ to $\mathrm{C}_{\mathrm{Glc}^{-}}-6(\delta \mathrm{c} 68.3)$, and from $\mathrm{H}_{\mathrm{Glc}^{\prime}}-1\left(\delta_{\mathrm{H}} 4.89\right)$ to $\mathrm{C}_{\mathrm{Rha}^{-}}-4(\delta \mathrm{c} 85.7)$, adequately illustrated the structure of 3 as $3 \beta$-hydroxy-23-acetyloxy-olean-12-en-28-oic acid 28-O- $\beta$-D-xylopyranosyl $(1 \rightarrow 6)$-[ $\beta$-D-glucopyranosyl-( $1 \rightarrow 4)-\alpha$-L-rhamnopyranosyl$(1 \rightarrow 2)]$ - $\beta$-D-glucopyranoside, named Bodinioside U.

Compound 4 was obtained as white amorphous powder. It exhibited a quasi-molecular ion peak at $m / z 1205.5950[\mathrm{M}-\mathrm{H}]^{-}$in the negative HR-ESI-MS spectrum, suggesting the molecular formula $\mathrm{C}_{58} \mathrm{H}_{94} \mathrm{O}_{26}$, indicating 12 degrees of unsaturation. Besides one hydroxyl taking the place of an acetoxy group substituent on C-23, most NMR signals (1 and 2) of 4 were nearly identical to those of Bodinioside $\mathrm{H}$ [4]. Five anomeric $\mathrm{H}$-atom at $\delta_{\mathrm{H}} 6.38$ (br. s), $6.11(\mathrm{~d}, J=7.9 \mathrm{~Hz}), 5.15(\mathrm{~d}, J=7.6 \mathrm{~Hz}), 5.02(\mathrm{~d}, J=7.4 \mathrm{~Hz})$ and $4.85(\mathrm{~d}, J=7.4 \mathrm{~Hz})$ in the ${ }^{1} \mathrm{H}$ NMR spectrum were ascribed to D-xylose, D-glucose, and L-rhamnose, respectively, in combination with acid hydrolysis and GC analysis. The Xyl unit was assigned to C-3 $(\delta \mathrm{c} 82.1)$ of the aglycone on the basis of the long-range correlation between $\mathrm{H}-1\left(\delta_{\mathrm{H}} 5.02\right)$ of $\mathrm{Xyl}$ and C-3. Meanwhile, a series of $\mathrm{HMBC}$ correlations from $\mathrm{H}_{\mathrm{Glc}}-1\left(\delta_{\mathrm{H}} 6.11\right)$ to C-28 $(\delta \mathrm{c} 176.7)$, from $\mathrm{H}_{\mathrm{Rha}}-1\left(\delta_{\mathrm{H}} 6.38\right)$ to $\mathrm{C}_{\mathrm{Glc}^{-}}-2(\delta \mathrm{c} 77.6)$, from $\mathrm{H}_{\mathrm{Xyl}^{\prime}}-1\left(\delta_{\mathrm{H}} 4.85\right)$ to $\mathrm{C}_{\mathrm{Glc}}-6(\delta \mathrm{c}$ 68.6), and from $\mathrm{H}_{\mathrm{Glc}^{\prime}}-1\left(\delta_{\mathrm{H}} 5.15\right)$ to $\mathrm{C}_{\mathrm{Rha}}-4(\delta \mathrm{c} 85.6)$ clarified the linkage of Glc, $\mathrm{Xyl}$ and Rha units at C-28 as shown. The structure of compound 4 was, therefore, concluded to be 3-O- $\beta$-D-xylopyranosyl-23-hydroxy-olean-12-en-28-oic acid 28-O- $\beta$-D- xylopyranosyl$(1 \rightarrow 6)$-[ $\beta$-D-glucopyranosyl-( $1 \rightarrow 4)$ - $\alpha$-L-rhamnopyranosyl-( $1 \rightarrow 2)]-\beta$-D- glucopyranoside, and named Bodinioside $\mathrm{V}$.

The molecular formula of Bodinioside W (5) was established as $\mathrm{C}_{52} \mathrm{H}_{84} \mathrm{O}_{21}$ on the basis of the negative HR-ESI-MS from the quasi molecular ion peak at $m / z 1089.5454$ [M $+\mathrm{COOH}]^{-}$, indicating 11 degrees of unsaturation. In the ${ }^{1} \mathrm{H}$ NMR spectrum of 5 , four anomeric H-atom at $\delta_{\mathrm{H}} 6.51(\mathrm{br} . \mathrm{s}), 6.11(\mathrm{~d}, J=8.0 \mathrm{~Hz}), 5.01(\mathrm{~d}, J=7.8 \mathrm{~Hz})$ and $4.85(\mathrm{~d}$, $J=7.4 \mathrm{~Hz}$ ) correlated with anomeric carbon signals at $\delta_{\mathrm{C}} 101.3,94.6,106.7$ and 105.4 in the HSQC spectrum, respectively. Acid hydrolysis of 5 yielded two D-xylose (Xyl), L-rhamnose (Rha) and D-glucose (Glc) as sugar residues by GC chromatography. Interpretation of its NMR data (Tables 1 and 2) revealed that the structure of compound $\mathbf{5}$ was closely related to compound 4, except for the presence of an additional Glc at C-28 of 4 . Thus, the structure of compound 5 was 3-O- $\beta$-D-xylopyranosyl-23-hydroxy-olean-12-en-28-oic acid 28 -O- $\beta$-D-xylopyranosyl- $(1 \rightarrow 6)$-[ $\alpha$-L-rhamnopyranosyl- $(1 \rightarrow 2)]-\beta$-D-glucopyranoside, and named Bodinioside $\mathrm{W}$. 
Compound 6 was obtained as white amorphous powder. It exhibited a quasi-molecular ion peak at $m / z 835.4459[\mathrm{M}+\mathrm{Na}]^{+}$in the positive HR-ESI-MS spectrum, suggesting the molecular formula of $\mathrm{C}_{42} \mathrm{H}_{68} \mathrm{O}_{15}$, indicating nine degrees of unsaturation. The ${ }^{1} \mathrm{H}$ NMR spectrum (Tables 1 and 2) of compound 6 showed two signals for anomeric protons at $\delta_{\mathrm{H}}$ $6.60(1 \mathrm{H}$, br. s) and $6.23(1 \mathrm{H}, \mathrm{d}, J=8.1 \mathrm{~Hz})$, which correlated with anomeric carbon signals at $\delta_{\mathrm{C}} 101.6$ and 95.1 in the HSQC spectrum, respectively, suggesting the presence of two sugar moieties. Acid hydrolysis of 6 yielded L-rhamnose (Rha) and D-glucose (Glc) as sugar residues by GC chromatography. The NMR data of $\mathbf{6}$ were highly analogous to the sericoside [13], suggested that they had same 2, 3, 19, 23-tetrahydroxy-olean-12-en-28-oic acid as the aglycone, except for the presence of an additional Rha at C-28 of 6 . The HMBC correlations from $\mathrm{H}_{\mathrm{Glc}}-1\left(\delta_{\mathrm{H}} 6.23\right)$ to $\mathrm{C}-28$, and from $\mathrm{H}_{\mathrm{Rha}}-1\left(\delta_{\mathrm{H}} 6.60\right)$ to $\mathrm{C}_{\mathrm{Glc}}-2(\delta \mathrm{c} 75.7)$, adequately illustrated the structure of 6 as $2 \alpha, 3 \beta, 19 \alpha, 23$ - tetrahydroxy-olean-12-en-28-oic acid 28-O- $\alpha$-L-rhamnopyranosyl-( $1 \rightarrow 2)-\beta$-D- glucopyranoside, named Bodinioside $X$.

Compound 7 was obtained as white amorphous powder. It exhibited a quasi-molecular ion peak at $m / z 835.4456[\mathrm{M}+\mathrm{Na}]^{+}$in the positive HR-ESI-MS spectrum, suggesting a molecular formula $\mathrm{C}_{42} \mathrm{H}_{68} \mathrm{O}_{15}$, indicating nine degrees of unsaturation. The ${ }^{1} \mathrm{H}$ NMR spectrum (Tables 1 and 2) of 7 revealed six methyl signals at $\delta_{\mathrm{H}} 1.13(3 \mathrm{H}, \mathrm{s}), 1.39(3 \mathrm{H}, \mathrm{s})$, $1.03(3 \mathrm{H}, \mathrm{s}), 1.19(3 \mathrm{H}, \mathrm{s}), 1.62(3 \mathrm{H}, \mathrm{s})$, and $1.06(3 \mathrm{H}, \mathrm{s})$ in correlation with carbons at $\delta_{\mathrm{C}}$ 14.2 (C-24), 17.5 (C-25), 17.4 (C-26), 24.1 (C-27), 26.9 (C-29), and 16.6 (C-30) in the HSQC spectrum, respectively. The signal at $\delta_{\mathrm{H}} 5.55(1 \mathrm{H}, \mathrm{br}$. s), corresponding to the carbon at $\delta_{\mathrm{C}} 128.2$ (C-12), coupled with $\delta_{\mathrm{C}} 139.2$ (C-13) in the ${ }^{13} \mathrm{C}$ NMR spectrum. On the basis of the above spectroscopic data, compound 7 was suggested to possess an ursane-12-ene skeleton [6]. Comparison of its NMR spectroscopic data with those of niga-ichigoside F1 [14], suggested that they had the same 2, 3, 19, 23-tetrahydroxy-urs-12-en-28-oic acid as the aglycone. Detailed comparison of ${ }^{1} \mathrm{H}$ and ${ }^{13} \mathrm{C}$ NMR spectral data of 7 with those of niga-ichigoside F1 indicated that they are highly structurally similar, except for the presence of an additional Rha at C-28 of $\mathbf{7}$. Acid hydrolysis of $\mathbf{7}$ yielded D-glucose (Glc) and L-rhamnose (Rha) as sugar residues by GC chromatography. The structure of compound 7 was, therefore, concluded to be $2 \alpha, 3 \beta, 19 \alpha, 23$-tetrahydroxy-urs-12-en-28-oic acid 28-O- $\alpha$-L-rhamnopyranosyl- $(1 \rightarrow 2)$ - $\beta$-D-glucopyranoside, and named Bodinioside $\mathrm{Y}$.

The anti-influenza A virus activity of compounds 1-7 against strain A/WSN/33/2009 was evaluated in MDCK cells, and their cytotoxicity was measured in parallel with the determination of antiviral activity, using oseltamivir as a positive control. It was found that compounds 1-7 displayed no significant cytotoxicity at $50 \mu \mathrm{M}$ concentration. Then, under this concentration, the in vitro potential anti-influenza A virus effects of all isolates were investigated. The results showed that compounds $\mathbf{2}$ and $\mathbf{5}$ exhibited moderate inhibition of influenza virus activities with inhibition rates of $35.33 \%$ and $24.08 \%$, while the inhibition rate of the positive control (oseltamivir) was $71.20 \%$.

\section{Discussion}

In summary, seven new triterpenoid saponins, including six oleanane triterpenoid saponins and a ursane one, named Bodiniosides S-Y, were isolated from the aerial parts of Elsholtzia bodinieri. Elucidation of their structures was performed based on extensive spectroscopic analyses. The anti-influenza activities of the isolates against A/WSN/33/2009 (H1N1) virus were investigated. The results demonstrated that compounds 2 and $\mathbf{5}$ exhibited potent inhibition of influenza virus activities, with inhibition rates of $35.33 \%$ and $24.08 \%$; meanwhile, compounds 1, 3, 4, 6 and 7 were found to be inactive, with an inhibition rate lower than $10 \%$. A previous study revealed that pentacyclic triterpenoids, including ursane, oleanane, and lupane types, have anti-influenza virus activity [15]. Our results further confirmed that the pentacyclic triterpenoids were active against influenza virus. This investigation should provide valuable information for further understanding of E. bodinieri. 


\section{Materials and Methods}

\subsection{General Experimental Procedures}

Optical rotations were recorded using a Jasco DIP-370 digital polarimeter (Jasco, Tokyo, Japan). UV spectra were performed on a UV-210A spectrophotometer (Shimadzu, Kyoto, Japan). IR spectra were obtained on a Bio-Rad FtS-135 spectrophotometer (Bio-Rad Laboratories, California, CA, USA) with KBr pellets. The 1D- and 2D NMR spectra were run on Bruker DRX-600 instruments (Bruker BioSpin Group, Rheinstetten, Germany) with TMS as an internal standard. ESI-MS and HR-ESI-MS were measured with an API-QstarTOF instrument (Applied Biosystem/MSD Sciex, Concord, ON, Canada). GC analysis was taken on Agilent Technologies HP5890 gas chromatograph (Agilent Technologies Inc., Massy, France) with flame ionization detector. Semi-preparative HPLC was run on an Agilent 1200 liquid chromatograph (Agilent Technologies Inc., Palo Alto, CA, USA) with a ZORBAX SB-C18 (5 A $\left.{ }^{\circ}, 9.4 \times 250 \mathrm{~mm}\right)$ column. Column chromatography $(\mathrm{CC})$ was carried out on silica gel (200-300 mesh, 80-100 mesh, Qingdao Marine Chemical Factory, Qingdao, China), Diaion HP-20SS (63-150 mm, Mitsubishi Fine Chemical Industries Co., Ltd., Tokyo,

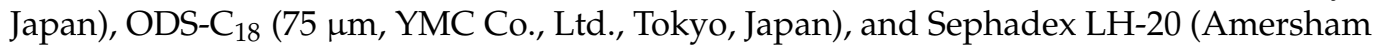
Biosciences AB, Uppsala, Sweden); thin-layer chromatography (TLC) was monitored by TLC plates (Si gel GF 254 , Qingdao Marine Chemical Factory, Qingdao, China), and spots were visualized by spraying with $5 \% \mathrm{H}_{2} \mathrm{SO}_{4}-\mathrm{EtOH}$, followed by heating on a hot plate. The purity (>95\%) of compounds 1-7 was determined by HPLC.

\subsection{Materials}

The aerial parts of E. bodinieri were collected in Yuxi city, Yunnan Province, P. R. China, in May 2016, and identified by Dr. Jindong Zhong. A voucher specimen (KMUST 20160005) was deposited at the Laboratory of Phytochemistry, Faculty of Life Science and Technology, Kunming University of Science and Technology.

\subsection{Extraction and Isolation}

The powered air-dried aerial parts of E. bodinieri $(15 \mathrm{~kg})$ were extracted with $75 \%$ aq. $\mathrm{Me}_{2} \mathrm{CO}(3 \times 35 \mathrm{~L}, 24 \mathrm{~h}$, each $)$ at room temperature and filtered. The filtrate was concentrated in vacuo, and the resulting residue was extracted successively with $\mathrm{CHCl}_{3}$, AcOEt and $n-\mathrm{BuOH}$, respectively.

The $n$ - $\mathrm{BuOH}$ extract $(300.0 \mathrm{~g})$ was separated over macroporous resin CC (Diaion HP-20SS) eluting with $\mathrm{MeOH} / \mathrm{H}_{2} \mathrm{O}$ (gradient30, 60, 90, and $100 \%$, each $15 \mathrm{~L}$ ) to obtain four fractions ( $F r$. A-D). Fr. C (eluted with $60 \% \mathrm{MeOH} / \mathrm{H}_{2} \mathrm{O}, 86.5 \mathrm{~g}$ ) was chromatographed successively over Sephadex LH-20 gel column $(20 \%, 30 \%, 40 \%, 50 \%, 60 \%$, and $100 \%$ $\mathrm{MeOH} / \mathrm{H}_{2} \mathrm{O}$, each $8 \mathrm{~L}$ ) to obtain subfractions Fr. C-1-C-6. Fr. C-1 (31 g) was isolated by ODS CC (eluted with $10 \%, 30 \%, 60 \%$, and $100 \% \mathrm{MeOH} / \mathrm{H}_{2} \mathrm{O}$ ) to obtain subfractions Fr. C-1-1-C-1-4. Fr. C-1-3 (13 g) was chromatographed over silica gel CC (eluted with $\mathrm{CHCl}_{3} / \mathrm{MeOH}$ 15: 1 to 0: 1$)$ to yield Fr. C-1-3-1-C-1-3-5. Compounds $1\left(t_{R}=15.0 \mathrm{~min}\right.$, $5.6 \mathrm{mg})$ and $2\left(t_{R}=20.1 \mathrm{~min}, 6.2 \mathrm{mg}\right)$ were purified from Fr. C-1-3-4 $(167 \mathrm{mg})$ via semipreparative HPLC $(58 \% \mathrm{MeOH}, 3 \mathrm{~mL} / \mathrm{min})$. Compounds $3\left(t_{R}=17.4 \mathrm{~min}, 4.3 \mathrm{mg}\right)$ and $6\left(t_{R}=22.1 \mathrm{~min}, 4.8 \mathrm{mg}\right)$ were purified from $F r$. C-1-3-3 $(103 \mathrm{mg})$ via semi-preparative HPLC ( $56 \% \mathrm{MeOH}, 3 \mathrm{~mL} / \mathrm{min})$. Compound $4\left(t_{R}=14.6 \mathrm{~min}, 5.6 \mathrm{mg}\right)$ was purified from $\mathrm{Fr}$. C-1-3-2 (84 mg) via semi-preparative HPLC (52\% MeOH, $3 \mathrm{~mL} / \mathrm{min})$. Fr. C-1-4 (2.5 g) was chromatographed over silica gel CC (eluted with $\mathrm{CHCl}_{3} / \mathrm{MeOH} 10: 1$ to $0: 1$ ) to yield $F r$. C-1-4-1-C-1-4-3. Compounds $5\left(t_{R}=15.6 \mathrm{~min}, 4.8 \mathrm{mg}\right)$ and $7\left(t_{R}=17.6 \mathrm{~min}, 7.3 \mathrm{mg}\right)$ were purified from $F r$. C-1-4-2 (163 mg) via semi-preparative HPLC ( $55 \% \mathrm{MeOH}, 3 \mathrm{~mL} / \mathrm{min}$ ).

\subsubsection{Bodinioside S (1)}

White amorphous powder. $[\alpha]_{\mathrm{D}}^{26.3}=-30.43(c=0.23, \mathrm{MeOH})$, IR $(\mathrm{KBr}): 3441,2942$, 1722, 1635, 1384, 1255, $1045 \mathrm{~cm}^{-1}$. UV $\lambda_{\max }(\mathrm{MeOH}) \mathrm{nm}(\log \varepsilon): 204$ (2.8). ESI-MS (neg.) $m / z: 1277[\mathrm{M}-\mathrm{H}]^{-}$, HR-ESI-MS (neg.) $m / z: 1277.6148[\mathrm{M}-\mathrm{H}]^{-}$, (Calcd. for $\mathrm{C}_{61} \mathrm{H}_{98} \mathrm{O}_{28}$, 1278.6245). ${ }^{1} \mathrm{H}$ and ${ }^{13} \mathrm{C}$ NMR: Tables 1 and 2. 


\subsubsection{Bodinioside $\mathrm{T}(2)$}

White amorphous powder. $[\alpha]_{\mathrm{D}}^{21.7}=-11.95(c=0.15, \mathrm{MeOH})$, ESI-MS (neg.) $m / z: 1247$ $[\mathrm{M}-\mathrm{H}]^{-}$, HR-ESI-MS (neg.) m/z: $1247.6070[\mathrm{M}-\mathrm{H}]^{-}$, (Calcd. for $\left.\mathrm{C}_{60} \mathrm{H}_{96} \mathrm{O}_{27}, 1248.6139\right)$. ${ }^{1} \mathrm{H}$ and ${ }^{13} \mathrm{C}$ NMR: Tables 1 and 2.

\subsubsection{Bodinioside U (3)}

White amorphous powder. $[\alpha]_{\mathrm{D}}^{21.8}=-8.24(c=0.34, \mathrm{MeOH})$, ESI-MS (pos.) $m / z: 1140$ $[\mathrm{M}+\mathrm{Na}]^{+}$, HR-ESI-MS (pos.) $\mathrm{m} / z: 1139.5614[\mathrm{M}+\mathrm{Na}]^{+},\left(\right.$Calcd. for $\left.\mathrm{C}_{55} \mathrm{H}_{88} \mathrm{O}_{23}, 1116.5716\right)$. ${ }^{1} \mathrm{H}$ and ${ }^{13} \mathrm{C}$ NMR: Tables 1 and 2.

\subsubsection{Bodinioside V (4)}

White amorphous powder. $[\alpha]_{\mathrm{D}}^{26.4}=-23.17(c=0.12, \mathrm{MeOH}), \mathrm{ESI}-\mathrm{MS}($ pos. $) \mathrm{m} / z: 1230$ $[\mathrm{M}+\mathrm{Na}]^{+}$, HR-ESI-MS (neg.) $\mathrm{m} / \mathrm{z}: 1205.5950[\mathrm{M}-\mathrm{H}]^{-},\left(\right.$Calcd. for $\left.\mathrm{C}_{58} \mathrm{H}_{93} \mathrm{O}_{26}, 1205.5955\right)$. ${ }^{1} \mathrm{H}$ and ${ }^{13} \mathrm{C}$ NMR: Tables 1 and 2.

\subsubsection{Bodinioside W (5)}

White amorphous powder. $[\alpha]_{\mathrm{D}}^{26.5}=-23.01(c=0.23, \mathrm{MeOH})$, ESI-MS (neg.) $\mathrm{m} / z$ : $1044[\mathrm{M}-\mathrm{H}]^{-}$, HR-ESI-MS (pos.) $\mathrm{m} / z: 1089.5454[\mathrm{M}+\mathrm{COOH}]^{-},\left(\right.$Calcd. for $\mathrm{C}_{52} \mathrm{H}_{84} \mathrm{O}_{21}$, 1044.5505). ${ }^{1} \mathrm{H}$ and ${ }^{13} \mathrm{C}$ NMR: Tables 1 and 2.

\subsubsection{Bodinioside X (6)}

White amorphous powder. $[\alpha]_{\mathrm{D}}^{24.6}=-20.43(c=0.16, \mathrm{MeOH})$, ESI-MS (pos.) $m / z: 835$ $[\mathrm{M}+\mathrm{Na}]^{+}$, HR-ESI-MS (pos.) $m / z: 835.4459[\mathrm{M}+\mathrm{Na}]^{+}$, (Calcd. for $\left.\mathrm{C}_{42} \mathrm{H}_{68} \mathrm{O}_{15}, 812.4558\right)$. ${ }^{1} \mathrm{H}$ and ${ }^{13} \mathrm{C}$ NMR: Tables 1 and 2.

\subsubsection{Bodinioside $Y(7)$}

White amorphous powder. $[\alpha]_{\mathrm{D}}^{24.2}=-10.69(c=0.26, \mathrm{MeOH})$, ESI-MS (pos.) $m / z: 835$ $[\mathrm{M}+\mathrm{Na}]^{+}$, HR-ESI-MS (pos.) $m / z: 835.4456[\mathrm{M}+\mathrm{Na}]^{+}$, (Calcd. for $\mathrm{C}_{42} \mathrm{H}_{68} \mathrm{O}_{15}, 812.4558$ ). ${ }^{1} \mathrm{H}$ and ${ }^{13} \mathrm{C}$ NMR: Tables 1 and 2.

\subsection{Acid Hydrolysis for Sugar Analysis}

Compounds 1-7 (1.0 mg for each compound) were hydrolyzed with $1 \mathrm{M} \mathrm{HCl}(0.4 \mathrm{~mL})$ and heated at $90-100{ }^{\circ} \mathrm{C}$ for $5 \mathrm{~h}$. The mixture was neutralized by the addition of Amberlite IRA400 $\left(\mathrm{OH}^{-}\right.$form $)$and then filtered. The filtrate was dried in vacuo, dissolved in $0.2 \mathrm{~mL}$ of pyridine containing $L$-cysteine methyl ester $(10 \mathrm{mg} / \mathrm{mL})$ and reacted at $60^{\circ} \mathrm{C}$ for $1 \mathrm{~h}$. To this mixture, a solution $(0.2 \mathrm{~mL})$ of trimethylsilyl imidazole in pyridine $(10 \mathrm{mg} / \mathrm{mL})$ was added, and then heated at $60{ }^{\circ} \mathrm{C}$ for $1 \mathrm{~h}$. The final mixture was directly analyzed by GC [30QC2/AC-5 quartz capillary column $(30 \mathrm{~m} \times 0.32 \mathrm{~mm})$ with the following conditions: column temperature: $180 / 280^{\circ} \mathrm{C}$; programmed increase $3{ }^{\circ} \mathrm{C} / \mathrm{min}$; carrier gas: $\mathrm{N}_{2}(1 \mathrm{~mL} / \mathrm{min})$; injection and detector temperature: $250{ }^{\circ} \mathrm{C}$; injection volume: $4 \mu \mathrm{L}$; split ratio: $1 / 50]$. The authentic samples $\mathrm{D}$ - and L-glucose, $\mathrm{D}$ - and L-xylose, $\mathrm{L}$-arabinose, and L-rhamnose were treated in the same manner. Under these conditions, the retention times of authentic samples D- and L-glucose, D- and L-xylose, L-arabinose and L-rhamnose were $18.29,18.87,13.35,14.01,14.30$ and $14.97 \mathrm{~min}$, respectively. During our studies, identical retention times observed between the different hydrolysates and authentic standards.

\subsection{Anti-Influenza Virus Activity}

The anti-influenza virus activities of compounds 1-7 were evaluated, using influenza strain A/WSN/33/2009 (H1N1). For the inhibitory activity assays, compounds 1-7 were dissolved and then diluted with DMSO, using Oseltamivir as a positive control. MDCK cells were seeded into 96-well plates, incubated overnight and infected with influenza virus (MOI $\frac{1}{4}$ 0.1). The cells were suspended in DMEM supplemented with $1 \%$ fetal bovine serum (FBS), containing test compound and $2 \mathrm{mg} / \mathrm{mL}$ TPCK-treated trypsin, and a final 
DMSO concentration of $1 \%$ was added in each well. After $40 \mathrm{~h}$ of incubation, Cell Titer-Glo reagent was added, and the plates were read, using a plate reader [15]. The inhibition rate was calculated by the following formula: inhibition rate $(\%)=[1-$ (luminescence with compounds - luminescence with compounds and virus)/(luminescence with DMSO luminescence with DMSO and virus)] $\times 100 \%$. Assessment of anti-influenza virus activity was performed as described previously [16].

Supplementary Materials: The following are available online. The IR, HR-ESI-MS, and 1D and 2D NMR spectra of the seven compounds are available in the supplementary materials.

Author Contributions: L.Y., J.D., R.L. and J.Z. conceived and designed the experiments; L.Y. and J.D. isolated the compounds; L.Y., J.D. and J.Z. elucidated the structures; F.Y. carried out the biological assay and helped with the preparation of the manuscript; L.Y., J.D. and J.Z. wrote the paper; and J.Z. managed the research project. All authors have read and agreed to the published version of the manuscript.

Funding: This research was funded by the grants of National Natural Science Foundation of China (grant number 31660100, U1602222), Yunnan Province applied Basic research project (grant number 2017BF034).

Institutional Review Board Statement: Not applicable.

Informed Consent Statement: Not applicable.

Data Availability Statement: Not applicable.

Conflicts of Interest: The authors declare no conflict of interest.

Sample Availability: Samples of compounds 1-7 are available from the authors.

\section{References}

1. Jiangsu New Medical College. Dictionary of Traditional Chinese Medicine; Shanghai Science and Technology Publishing House: Shanghai, China, 1985; 489p.

2. Li, H.Z.; Fu, L.Z.; Li, H.M.; Li, R.T.; Deng, X.L. Two new oleanane triterpenoid saponins from Elsholtzia bodinieri. Phytochem. Lett. 2012, 5, 572-575. [CrossRef]

3. Zhao, X.W.; Zhong, J.D.; Li, H.M.; Li, R.T. Three new 18, 19-seco-ursane glycosides from Elsholtzia bodinieri. Phytochem. Lett. 2015, 12, 308-312. [CrossRef]

4. Zhong, J.D.; Zhao, X.W.; Li, H.M.; Gao, L.H.; Li, R.T. Five new oleanane triterpenoid saponins from the aerial parts of Elsholtzia bodinieri. Helv. Chim. Acta 2016, 99, 204-209. [CrossRef]

5. Zhong, J.D.; Zhao, X.W.; Chen, X.Q.; Li, H.M.; Chen, C.H.; Xia, X.S.; Li, R.T. Two new ursane-type triterpenoid saponins from Elsholtzia bodinieri. Arch. Pharm. Res. 2016, 39, 771-777. [CrossRef] [PubMed]

6. Xiang, L.L.; Zhang, L.; Chen, X.Q.; Xia, X.S.; Li, R.T.; Zhong, J.D. Ursane-type triterpenoid saponins from Elsholtzia bodinieri. Nat. Prod. Res. 2018, 33, 1349-1356. [CrossRef] [PubMed]

7. Li, R.T.; Li, J.T.; Wang, J.K.; Han, Q.B.; Zhu, Z.Y.; Sun, H.D. Three new flavonoid glycosides isolated from Elsholtzia bodinieri. Chem. Pharm. Bull. 2008, 56, 592-594. [CrossRef] [PubMed]

8. Zhong, J.D.; Feng, Y.; Li, H.M.; Li, H.Z.; Xia, X.S.; Li, R.T. A new flavonoid glycoside from Elsholtzia bodinieri. Nat. Prod. Res. 2016, 30, 2278-2284. [CrossRef] [PubMed]

9. Hu, H.B.; Jian, Y.F.; Zheng, X.D.; Cao, H. Three sesquiterpene glycosides from Elsholtzia bodinieri. Bull. Korean Chem. Soc. 2007, 28, 467-470. [CrossRef]

10. Hu, H.B.; Cao, H.; Jian, Y.F.; Zheng, X.D.; Liu, J.X. Two new clerodane diterpenoid glucosides and other constituents from the roots of Elsholtzia bodinieri Van't. Indian J. Chem. B. 2008, 47B, 166-170.

11. Hu, H.B.; Jian, Y.F.; Cao, H.; Zheng, X.D. Phenolic compounds from Elsholtzia bodinieri Van't. J. Chin. Chem. Soc. 2007, 54, 1189-1194. [CrossRef]

12. Mohammad, F.V.; Noorwala, M.; Ahmad, V.U.; Sener, B. A bidesmosidic hederagenin hexasaccharide from the roots of Symphytum officinale. Phytochemistry 1995, 40, 213-218. [CrossRef]

13. Ponou, B.K.; Teponno, R.B.; Ricciutelli, M.; Quassinti, L.; Bramucci, M.; Lupidi, G.; Barboni, L.; Tapondjou, L.A. Dimeric antioxidant and cytotoxic triterpenoid saponins from Terminalia ivorensis A. Chev. Phytochemistry 2010, 71, 2108-2115. [CrossRef] [PubMed]

14. Nam, J.H.; Jung, H.J.; Tapondjou, L.A.; Lee, K.T.; Choi, J.H.; Kim, W.B.; Park, H.J. The anti-hyperlipidemic effects and constituents of the 19 $\alpha$-hydroxyursane-type triterpenoid fraction obtained from the leaves of Rubus crataegifolius. Nat. Prod. Sci. 2007, 13, 152-159. 
15. Wang, H.; Xu, R.Y.; Shi, Y.Y.; Si, L.L.; Jiao, P.X.; Fan, Z.B.; Han, X.; Wu, X.Y.; Zhou, X.S.; Yu, F.; et al. Design, synthesis and biological evaluation of novel L-ascorbic acid-conjugated pentacyclic triterpene derivatives as potential influenza virus entry inhibitors. Eur. J. Med. Chem. 2016, 110, 376-388. [CrossRef] [PubMed]

16. Song, W.; Si, L.L.; Ji, J.S.; Wang, H.; Fang, X.M.; Yu, L.Y.; Li, R.Y.; Liang, L.N.; Zhou, D.; Ye, M. Uralsaponins M-Y, antiviral triterpenoid saponins from the roots of Glycyrrhiza uralensis. J. Nat. Prod. 2014, 77, 1632-1643. [CrossRef] [PubMed] 